「材料」 (Journal of the Society of Materials Science, Japan), Vol. 70, No. 1, pp. 1-4, Jan. 2021

Original Papers

\title{
Effect of Gelation Concentration on Tensile and Stress Relaxation Behavior of $\kappa$-Carrageenan Hydrogels
}

by

\author{
Jun-ichi HORINAKA*, Kanji YAMAMOTO** and Toshikazu TAKIGAWA*
}

The uniaxial stretching and the stress relaxation measurements were carried out for $\kappa$-carrageenan hydrogels prepared at different gelation concentration $\left(c_{0}\right)$. The Young's modulus $(E)$ for the gel with low $c_{0}$ was greater than that for the gel with high $c_{0}$. Significant difference also appeared in the stress relaxation behavior: the gel with low $c_{0}$ showed greater and slower stress reduction. The results were explained by the specific network structure of $\kappa$-carrageenan hydrogels, where the crosslinks are composed of aggregates of $\kappa$-carrageenan chains. It was proposed that a great number of small crosslinks are generated at low $c_{0}$ including imperfect fragile crosslinks and that the weak crosslink domains disappear accompanying the uniaxial stretching of the gel. It seemed that the dissociation occurs successively under the tensile stress and contributes to the stress relaxation.

Key words:

к-Carrageenan, Hydrogel, Gelation concentration, Tensile behavior, Crosslink

\section{Introduction}

Polysaccharides are natural polymers composed of monosaccharide units linked via glycosidic bonds. A wide variety of natural products such as plants, seaweeds, and microorganisms contain specific polysaccharides. Nowadays polysaccharides have been used for foods, cosmetics, and medicines due to their biocompatibility. The fact that most water-soluble polysaccharides form hydrogels under appropriate conditions has been also utilized. $\kappa$-Carrageenan is one of such water-soluble polysaccharides consisting of repeating units of a 3,6-anhydro-galactose and a monosulfated galactose. $\kappa$-Carrageenan chains dissolved in aqueous solutions tend to transform from random-coil to helical conformation on cooling and furthermore the helical chains aggregate to form crosslinks of the hydrogels above the critical concentrations. Hence, the crosslinks of $\kappa$-carrageenan hydrogels are composed of aggregates of $\kappa$-carrageenan chains in the helical conformation, which are different from crosslinks of ordinary chemical and physical gels ${ }^{1)-3)}$.

Mechanical properties of $\kappa$-carrageenan hydrogels have been extensively studied by many research groups to develop industrial applications variously. Accordingly, effects of preparation conditions of $\kappa$-carrageenan hydrogels on the mechanical properties have been paid attention, wherein effects of the species and the concentration of additives like salts and sugars have been also examined ${ }^{4)-6)}$. On the other hand, there is little information on the effects of concentration of $\kappa$-carrageenan itself; specifically, effect of gelation concentration, the concentration of $\kappa$-carrageenan when the aqueous system becomes gel, remains to be examined.

In this study, mechanical properties have been compared between $\kappa$-carrageenan hydrogels prepared at different gelation concentrations. The uniaxial stretching and subsequent stress relaxation behavior has been measured. The $\kappa$-carrageenan concentration in the hydrogels has been adjusted to be almost the same regardless of the gelation concentration before the mechanical measurement. The difference in the tensile and the relaxation behavior of the $\kappa$ carrageenan hydrogels has been explained in terms of the role of the specific crosslinks.

\footnotetext{
$\dagger$ Received May 29, 2020 @2021 The Society of Materials Science, Japan

* Member: Dept. Mater. Chem., Kyoto Univ., Nishikyo-ku, Kyoto 615-8510, Japan

** Dept. Mater. Chem., Kyoto Univ., Nishikyo-ku, Kyoto 615-8510, Japan
} 


\section{Experimental}

\subsection{Sample preparation}

$\kappa$-Carrageenan (TCI, Japan), and an additive $\mathrm{KCl}$ (Wako, Japan) were used as received. Fig. 1 shows the chemical structure of $\kappa$-carrageenan; the metal content of the $\kappa$ carrageenan as the counter ion estimated by the inductively coupled plasma atomic emission spectroscopy is listed in Table 1 . The concentration of $\kappa$-carrageenan in the pre-gel aqueous solution, which is referred to as the gelation concentration $\left(c_{0}\right)$, was determined through preliminary gelation tests; the concentrations finally chosen in this study are shown in Table 2. The values of $c_{0}$ were set apart as far as possible within available gelation conditions. Practically, small amount of $\mathrm{KCl}(\mathrm{CKCl}=5 \mathrm{mM})$ was added to the hydrogels to obtain handleable gels. In the preliminary tests, gelation temperature $\left(T_{\mathrm{gel}}\right)$ was determined as follows: Pure aqueous solutions of $\kappa$-carrageenan and the additives were mixed together well at $80{ }^{\circ} \mathrm{C}$ and the gelation of the pre-gel solutions was judged by the fluidity after being stored at a given temperature for $24 \mathrm{~h}$ in a water bath. The temperature of the water bath was stepped down by $1{ }^{\circ} \mathrm{C}$ and $T_{\text {gel }}$ was defined as $T_{\mathrm{c}}+0.5{ }^{\circ} \mathrm{C}$, where $T_{\mathrm{c}}$ was the highest gelation temperature on the fluidity basis. The obtained values of $T_{\text {gel }}$ are shown in Table 2. $\kappa$-Carrageenan hydrogels used for mechanical measurements were prepared in a similar manner. The pre-gel solution was stuffed in a rectangular mold made of metal and Teflon and then the mold was stored at $T_{\text {gel }}-5^{\circ} \mathrm{C}$ for $24 \mathrm{~h}$. The prepared hydrogels were placed on a Teflon plate allowing the solvent water to evaporate until the $\kappa$ carrageenan concentration in the hydrogels $(c)$ reached a value in the range of $50 \leq c \leq 100 \mathrm{gL}^{-1}$. Then the gels were cut into rectangular pieces with the dimensions of $10 \mathrm{~mm} \times 30 \mathrm{~mm} \times$ $3 \mathrm{~mm}$. In practice $c$ was estimated from the volume and the dry weight of the sample after the mechanical measurement.

Table 1 Metal content of $\kappa$-carrageenan used in this study.

\begin{tabular}{ccc}
\hline $\mathrm{Na}^{+}$ & $\mathrm{K}^{+}$ & $\mathrm{Ca}^{2+}$ \\
\hline 0.28 & 7.8 & 0.13 \\
\hline
\end{tabular}

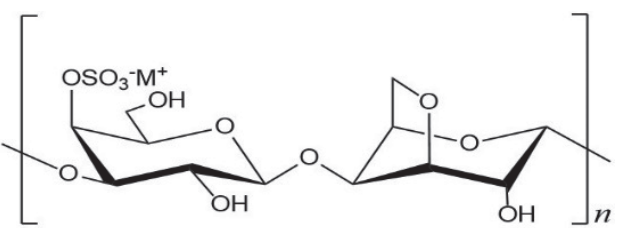

$\mathrm{M}=\mathrm{Na}, \mathrm{K}, \mathrm{Ca}, \mathrm{H}$

Fig. 1 Chemical structure of $\kappa$-carrageenan.

Table 2 Characteristics of samples.

\begin{tabular}{cccc}
\hline sample code & $c_{0} / \mathrm{gL}^{-1}$ & $c_{\mathrm{KCl}} / \mathrm{mM}$ & $T_{\text {gel }} /{ }^{\circ} \mathrm{C}$ \\
\hline $\mathrm{C} 05$ & 5 & 5 & 11.5 \\
$\mathrm{C} 30$ & 30 & 5 & 51.5 \\
\hline
\end{tabular}

\subsection{Measurement}

Tensile stretching and stress relaxation measurements for the $\kappa$-carrageenan hydrogels were carried out with a commercial tensile machine (TENSILON RTM-250, Orientec, Japan). The measurement temperature was $25{ }^{\circ} \mathrm{C}$. The hydrogel samples were uniaxially stretched between cramps at a constant speed of $1 \mathrm{~mm} / \mathrm{min}$. The initial length between the cramps was around $15 \mathrm{~mm}$. In the stress relaxation measurement, the initial strain of $1 \%$ was applied to the sample at the strain rate of $10 \mathrm{~mm} / \mathrm{min}$, so that an instant deformation could be assumed. The stress $(\sigma)$ was calculated from the force and the cross section of the gel sample measured before stretching; hence, $\sigma$ obtained in this study was the nominal tensile stress.

\section{Results and Discussion}

Fig. 2 shows the stress-strain curves for $\mathrm{C} 05$ at $c=$ $74 \mathrm{gL}^{-1}$ and $\mathrm{C} 30$ at $c=95 \mathrm{gL}^{-1}$. It is obvious that the stressstrain behavior depends much on $c_{0}$; each stress-strain curve appears almost linear within the $\varepsilon$-range examined but the slope of the curve, which is defined as the Young's modulus $(E)$, for $\mathrm{C} 05$ is obviously greater than that for C30. In fact, the values of $E$ for $\mathrm{C} 05$ and $\mathrm{C} 30$ estimated from Fig. 2 are $5.5 \times$ $10^{6}$ and $1.1 \times 10^{6} \mathrm{~Pa}$, respectively. Three independent tensile measurements have been carried out for $\mathrm{C} 05$ and $\mathrm{C} 30$ at the same $c$ to confirm the reproducibility of $E$. The obtained standard deviations for $E$ are $2.2 \times 10^{6} \mathrm{~Pa}$ for $\mathrm{C} 05$ and $0.31 \times$ 
$10^{6} \mathrm{~Pa}$ for $\mathrm{C} 30$. These results indicate that $\mathrm{C} 05$ gel is significantly stiffer than $\mathrm{C} 30$ gel in this $c$-range in terms of $E$.

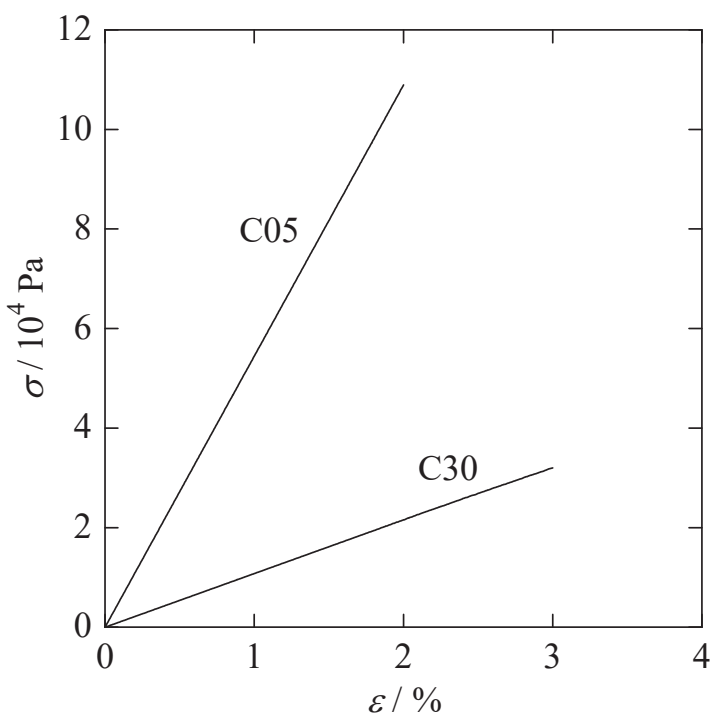

Fig. 2 Stress-strain curves for C05 and C30.

The stress relaxation behavior is compared between $\mathrm{C} 05$ and $\mathrm{C} 30$ in Fig. 3; actually, the relaxation modulus $(E(t))$ reduced by the initial value $E(0)$ is plotted. Here, $c$ for $\mathrm{C} 05$ and $\mathrm{C} 30$ samples are $c=52 \mathrm{gL}^{-1}$ and $50 \mathrm{gL}^{-1}$, and $E(0)$ are $6.5 \times 10^{5}$ and $1.5 \times 10^{5} \mathrm{~Pa}$, respectively. It is seen that the extent of the stress reduction for $\mathrm{C} 05$ is greater than that for $\mathrm{C} 30$. It also seems that the stress reduction for $\mathrm{C} 05$ continues until the end of measurement of $80 \mathrm{~s}$, while the stress for $\mathrm{C} 30$ becomes constant at about $30 \mathrm{~s}$.

The difference in the tensile and stress relaxation behavior between C05 and C30 in Figs. 2 and 3 might be related to the properties of crosslink domains in the gels. As described in the introduction, the crosslinks of the hydrogels are composed of the aggregates of helices. This implies that the aggregation near the gelation point proceeds smoother for $\mathrm{C} 05$ than for $\mathrm{C} 30$ due to the lower solution viscosity, resulting in the larger number of crosslinks for $\mathrm{C} 05$. It still remains unclear what determines the modulus of the hydrogels, but the number of active chains (probably, the number of fibrils, in our case) must control the elastic modulus as is the chemical gels. Because the number of active fibrils can also read as the number of crosslinks, the higher value of $E$ for $\mathrm{C} 05$ can be explained by the difference in the number density of crosslink domains. In a fast gelation process, there might be generated rather weak crosslink domains, which experiences the breakdown in the course of tensile tests. This breakdown corresponds to the dissociation of helices and continues even after extension which emerges as a stress relaxation in Fig. 3. A large extent of stress relaxation for C05 shown in Fig. 3 can be attributed to a larger number of the weak crosslinks for C05. This may also produce the gradual decrease in modulus.

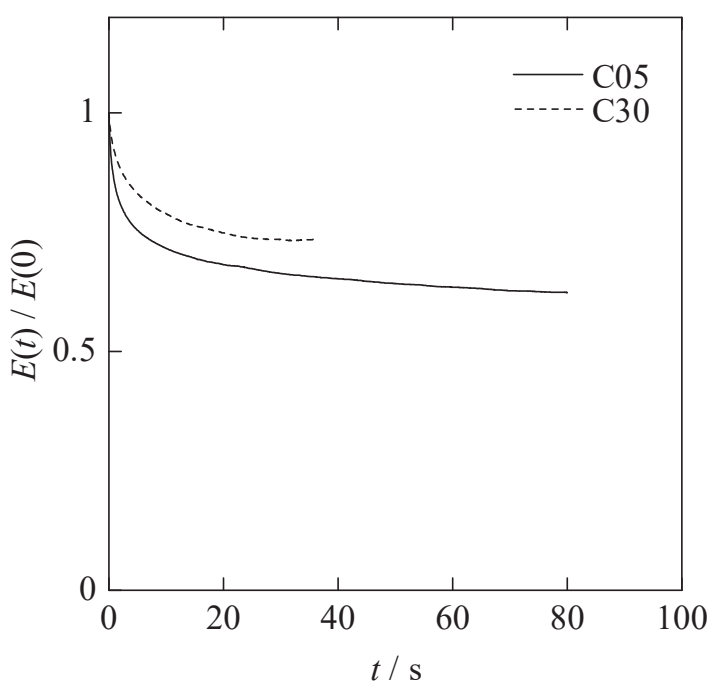

Fig. 3 Comparison of stress relaxation behavior between $\mathrm{C} 05$ and $\mathrm{C} 30$. $E(t)$ is reduced by the initial value.

The stress relaxation resulting from the breakdown of the weaker crosslinks is observed even in the repeated stretching of the $\kappa$-carrageenan hydrogels. Fig. 4 shows $E(t)$ for $\mathrm{C} 05$ and $\mathrm{C} 30$ obtained by repeated stretching measurements. The curves of run 1 are identical to those in Fig. 3. In the experiments, after a stress relaxation test was finished, the zero-strain state is recovered. Then, the next run was performed. Fig. 4(a) demonstrates that the trend of the stress relaxation for $\mathrm{C} 05$ characterized by the slower as well as larger relaxation is almost maintained in each run, but the $E(t)$ curve shifts to upper side as the number of repetition increases. The results for C30 demonstrate similar trend in Fig. 4(b), Concretely speaking, $E(0)$ at runs 1 through 3 for $\mathrm{C} 05$ are $6.5 \times 10^{5}, 7.0 \times 10^{5}$, and $7.5 \times 10^{5} \mathrm{~Pa}$, and $1.5 \times 10^{5}, 1.7 \times 10^{5}, 1.8 \times 10^{5} \mathrm{~Pa}$ for $\mathrm{C} 30$. 
Although we do not know the origin of the upper shift at present, this may be due to the orientation of fibrils by extension and the degree of orientation increases with increasing repetition of stretch.
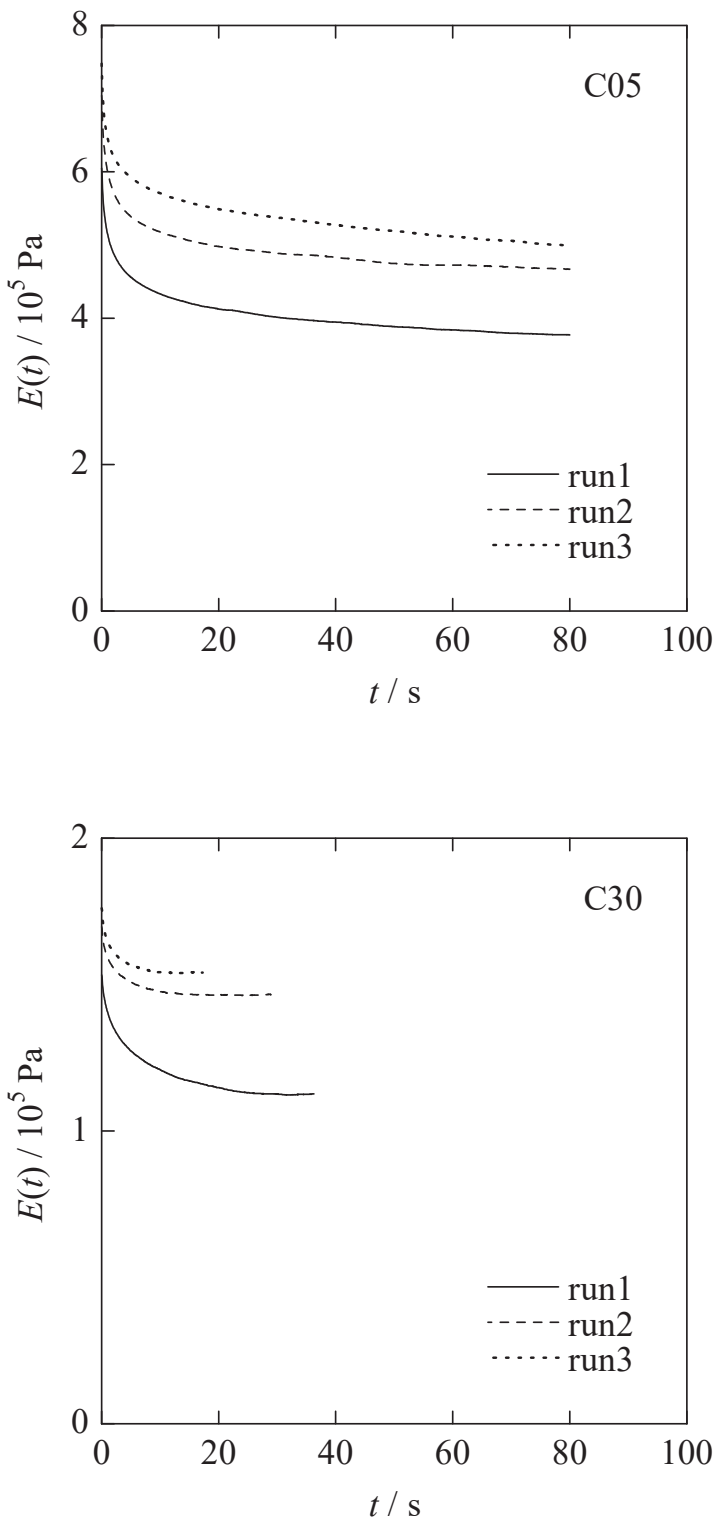

Fig. 4 Enhancement of $\mathrm{E}(t)$ by repetitive stress relaxation for (a) $\mathrm{C} 05$ (b) $\mathrm{C} 30$.

\section{Conclusions}

Tensile and stress relaxation behavior of $\kappa$-carrageenan hydrogels has been compared between C05 and C30. Both samples have been prepared via gelation of pre-gel solutions with concentrations set apart as far as possible and measured at almost the same $\kappa$-carrageenan concentration. $\mathrm{C} 05$ has exhibited greater $E$ than $\mathrm{C} 30$. In the stress relaxation, the stress reduction for $\mathrm{C} 05$ is greater and faster. These results have been explained by the difference in the number and the stability of the crosslinks in the network structure. The dissociation of the weak crosslink domains has been proposed.

\section{References}

1) E. R. Morris, D. A. Rees, D. A. and G. Robinson, "Cationspecific aggregation of carrageenan helices - domain model of polymer gel structure". Journal of Molecular Biology, vol. 138, pp. 349-362 (1980).

2) C. Viebke, L. Piculel and S. Nilsson, "On the mechanism of gelation of helix-forming biopolymers". Macromolecules, vol. 27, pp. 4160-4166 (1994).

3) S. Ikeda, V. J. Morris and K. Nishinari, "Microstructure of aggregated and nonaggregated kappa-carrageenan helices visualized by atomic force microscopy". Biomacromolecules, vol. 2, pp. 1331-1337 (2001).

4) M. Watase and K. Nishinari, "Rheological and thermal properties of agarose and kappa-carrageenan gels containing urea, guanidine hydrochloride or formamide". Food Hydrocolloids, vol. 1, pp. 25-36 (1986).

5) C. Rochas, M. Rinaudo and S. Landry, "Role of the molecular weight on the mechanical properties of kappacarrageenan gels". Carbohydrate Polymers, vol. 12, pp. 255-266 (1990).

6) G. Sason and A. Nussinovitch, "Characterization of $\kappa$ carrageenan gels immersed in ethanol solutions". Food Hydrocolloids, vol. 79, pp. 136-144 (2018). 